\title{
Urbanisation, dietary patterns and body composition changes in adolescent girls: a descriptive cross sectional study
}

\author{
Maduka de Lanerolle-Dias ${ }^{1}$, Pulani Lanerolle ${ }^{1}$, Sunethra Atukorala ${ }^{1}$ and Angela de Silva ${ }^{2^{*}}$
}

\begin{abstract}
Background: Urbanisation has been reported to have negative effects on lifestyle and possibly on body composition. The aim of this study was to assess the effect of urbanization on dietary patterns and measures of body composition in Sri Lankan adolescent girls.

Methods: Body Mass Index was calculated in 552 adolescent school dropouts. Percentage body fat was calculated using a population specific skin fold thickness equation. Dietary patterns were identified based on information obtained from a food frequency questionnaire. Extraction of factors of the dietary patterns was by principal component analysis. The sum of hours / week spent on physical activity was calculated. Focus group discussions were held to gather supportive qualitative data on food related behaviors. Multivariate linear regression models were used to assess the association between independent variables and dietary patterns.

Results: Two dietary patterns were identified; a convenience-based dietary pattern with higher factor loadings for starchy foods other than rice, animal foods, dairy products, fruits, sweetened food and fried food, and a traditional pattern with higher factor loadings for rice, tubers and potatoes, vegetables and dark green leafy vegetables. An urban girl had greater than three times the risk of having a higher percentage body-fat than a rural girl $(P=0.01$, $\mathrm{OR}=3.2)$. Time spent on physical activity was significantly $(P=0.003)$ higher among the rural girls. Focus group discussions indicate that urban girls preferred food from vendors due to convenience while rural girls preferred home cooked meals.
\end{abstract}

Conclusions: Urban adolescents in this study followed a convenience based dietary pattern and this dietary pattern and lower physical activity was associated with overweight and higher body fat.

Keywords: Adolescents, Urbanization, Dietary pattern, Principal component analysis

\section{Background}

The socio demographic transition in developing countries has resulted in an associated lifestyle transition which has adversely affected population health. Evidence of patterning studies in societies with changing demographic patterns show that urban diets and dietary patterns differ from rural ones in that, nutrition transition has resulted in a diet high in fat [1]. A study from China reported that while a traditional Chinese diet is low in

\footnotetext{
* Correspondence: desilva.angela@gmail.com

${ }^{2}$ Department of Physiology, Faculty of Medicine, University of Colombo, Kynsey road, Colombo 8, Sri Lanka

Full list of author information is available at the end of the article
}

fat, people in urban areas follow a dietary pattern with more than $30 \%$ of energy from fat [2].

Evidence also show that urbanization not only has a direct effect on a people's food consumption, behavior and lifestyle practices, in turn affecting their level of physical activity, sedentary behavior and diet but also body composition [3]. A study carried out on Indian children report two dietary patterns; 'snack and fruit' and 'lacto-vegetarian'. The snack and fruit pattern associated with the urban dwelling included sweetened drinks, branded noodles and, leavened breads, which were mostly purchased and the lacto vegetarian pattern associated with rural dwelling [4]. 
Principal component analysis carried out on dietary data of these children showed association with urban /rural dwelling, anthropometric profile and body composition. Studies in European children have identified that 'snacking' and 'energy dense' patterns are associated with overweight [5] and higher fat mass [6]. A similar study carried out on black and white adolescent girls report four dietary patterns where the healthy pattern, characterized by a high intake of fruits, vegetables, dairy, grains without added fats, mixed dishes and soups, and a low intake of sweetened drinks, other sweets, fried foods, burgers, and pizza, was related to the smallest increase in waist circumference[7].

The demographic transition from rural to urban areas that is ongoing in most low and middle income countries is thought to be accompanied by changes in dietary patterns which may have a negative impact on health and increase the risk of chronic diseases such as diabetes and cardio vascular disease. To date, there has been no data on dietary patterns in urban and rural Sri Lankan children, and their association with anthropometric profiles. Rapid changes in physical growth and psychosocial development place adolescents as a particularly vulnerable group to poor diet and nutrition in the setting of the nutrition transition. This is more so in children from urban, low income groups, who often drop out of school [8]. While adolescent diets tend to be lacking nutrient adequacy [9], adolescent school dropouts have been found to be even more vulnerable to nutritional setbacks [10]. The aim of this study was to observe the effect of urbanization, through the association between area of residence, with dietary patterns and body composition measures, and to relate these findings to life style factors and habits influencing dietary intake obtained through qualitative data in Sri Lankan female adolescent school dropouts.

\section{Methods}

The study was a descriptive cross sectional study and sample size was calculated using an equation by Fleiss, 1981 by considering $20 \%$ difference between the intervention and control groups in relation to knowledge on micronutrients, an alpha error of $5 \%$, beta error of $10 \%$ (power of $90 \%$ ) with 1:1 ratio of the two groups, where the minimum sample size required was calculated to be 550 . The sample was stratified by urban $(n=300)$ and rural sectors $(n=300)$, and a total of 600 participants were recruited.

According to available baseline data on the proportion of out of school adolescents in each area, six Public Health Midwife (PHM) areas were selected from each of these two districts and all school dropouts within each were recruited to the study. All participants were examined by a medical officer who excluded those with any known medical conditions. Exclusion criteria were pregnancy, lactation or having a child below the age of six months. Complete dietary data were available for 552 female adolescent (aged 15-19 years of age) school dropouts. All dietary, anthropometric, body composition and qualitative data included in the analysis are from the pre-intervention data and are not subject to bias due to intervention. This study was conducted according to the guidelines laid down in the declaration of Helsinki and all procedures involving human subjects were approved by the ethics review committee of the Faculty of Medicine, University of Colombo, Sri Lanka. Written informed consent was obtained from all study participants.

\section{Anthropometric data}

Weight was measured to the nearest $0.1 \mathrm{~kg}$ with a calibrated electronic scale (Seca813). Height was determined to the nearest $0.1 \mathrm{~cm}$ using a stadiometer (Seca225, telescopic height measurement) according to standard protocol. In order to avoid variability in results, all height and weight data were measured by one researcher using the same equipment and participants were attired in light indoor clothing. Body mass index (BMI) was calculated as weight $(\mathrm{kg}) /$ height $\left(\mathrm{m}^{2}\right)$, and categorized into underweight, normal weight and overweight on the basis of age specific World Health Organization classification of BMI for adolescent girls [11].

\section{Percentage body fat calculation}

A skin-fold thickness (SFT) equation [12] specific for 15-19 year old Sri Lankan adolescent girls was used for the calculation of percentage body fat. The triceps and supra-iliac skin fold thickness required for the equation was measured in triplicate on the left side of the body, in accordance with standard protocols [12], using a Harpenden caliper (gradation $0.2 \mathrm{~mm}$, range: $80 \mathrm{~mm}$, Seca, HSK-BI), to the nearest $1 \mathrm{~mm}$. The average of three SFT recordings was used in the analysis. Percentage body fat $(\mathrm{kg})$ was categorized into tertiles as low $(<12 \mathrm{~kg})$, moderate $(12-27 \mathrm{~kg})$ and excess $(>27 \mathrm{~kg})$ body fat.

\section{Dietary data}

An interviewer administered food frequency questionnaire was used for obtaining information regarding the weekly frequency of consumption of common food items. The questionnaire assessed the frequency of consumption and portion size of carbohydrates (breakfast, lunch and dinner), animal food, dairy, beverages, fruits and vegetables, sweets, snacks and instant food over a week (95 items). Portion size was determined using a pretested colored photographic atlas of selected cooked foods as well as by kitchen utensils where photos were not available (tablespoon, rice spoon, glass, tea cup).Weights of the cooked food were converted 
to the raw weights. The response options for each food item included never/rarely consumed, number of times consumed per day(1-3: breakfast, lunch, dinner) and number of days consumed per week (1-7).This food frequency questionnaire was pretested on 20 girls of similar age and area of residence in a pilot study.

\section{Physical activity}

Physical activity was assessed by obtaining data on the number of hours per day and days per week spent on walking, exercising and carrying out strenuous activities. The sum of hours / week spent on these (at home and at work) was used for analysis as physical activity per week. Physical activity/week was categorized in to tertiles as slow ( $<9 \mathrm{hrs} /$ week), medium (9-34 hrs/week) and high ( $>34$ hrs/week) physical activity.

\section{Qualitative data}

Separate focus group discussions were held with 15-19 year girls, their mothers, health volunteers and PHMs of the urban and rural areas. Discussions were on the following themes: foods consumed, preferences in diet, Cooking practices, perceived constraints to good nutrition and myths associated with nutrition and diet. Focus group discussions were held prior to carrying out other investigations, so as to avoid bias in the discussion, and were held until saturation point was reached. Sessions were hand written and also recorded for accuracy and data was cross checked by a second investigator for validity. The data collected from focus group discussions was classified according to identified themes and analyzed using the cut-and paste technique described by Stewart and Shamadasani and triangulated with quantitative data [13].

\section{Identification of dietary patterns}

Food items listed in the food frequency questionnaire were grouped into 17 food groups (Rice and rice based products, potatoes and tubers, vegetables, dark green leafy vegetables (DGLV), wheat flour based products, pulses, fresh fruits, pickled fruits with added sugar, fish, meat, processed meat, liver, egg, dairy, sweetened beverages, sweets and fried food) according to their nutritional characteristics. Dietary patterns were identified based on the frequency of consumption of each of the 17 food groups using exploratory factor analysis, which is an analytical tool. Factor scores (composite variables which provide information about an individual's placement on the factor(s)). were computed by the statistical package for each of the dietary patterns, and these factor scores were used in subsequent analysis. The Bartlett test of sphericity (BTS) and the Kaiser-Meyer-Olkin measure of sampling adequacy (KMO) were used to assess data adequacy for factor analysis. The factor analysis model would be considered appropriate with a KMO greater than 0.6 and BTS of $P<0.05$. A correlation matrix was then constructed. Principal component analysis was used for the extraction of factors. The first model was tested without setting the eigen value. The number of retainable factors was determined by the Cattel's Scree Plot method. Then, a second model was tested setting the eigen value to 1.5. Factors with eigen values greater than 1.5 were retained. Food groups with factor loadings greater than 0.30 and communality over 0.20 were retained in the patterns identified [14].

\section{Data analysis}

The Statistical Package for the Social Sciences for Windows (v. 18.0, SPSS Inc., Chicago, IL, USA) was used in the data analysis. Body fat was compared with BMI and diet. The independent samples $t$ test was used to assess the association between independent variables and factor score of the dietary patterns. BMI, body fat and physical activity of girls in urban and rural areas were compared using ANOVA, where BMI, physical activity and body fat were considered as dependent variables and area of residence was considered as the factor score. For the purpose of logistic regression, BMI and Fat mass were regrouped, where BMI was categorized as overweight and non overweight (by combining the underweight and normal weight), and fat mass was categorized as having excess body fat and low or moderate body fat (by combining the first and second tertiles). Logistic regression models were used to calculate the odds ratio. When identifying the likelihood of a girl residing in an urban area being overweight; and BMI (overweight vs. non overweight), Body fat (excess vs. low/moderate), physical activity(hrs/wk), and factor scores for pattern 1 and 2 were considered the independent variables when identifying the likelihood of an urban girl having excess body fat. Factor scores of the two patterns were compared with area of residence, BMI status and physical activity using ANOVA, Post Hoc tests were carried out where there were more than two categories in a variable (i.e. BMI status: underweight, normal weight, over weight and Physical activity: low medium, high). Multivariate linear regression models were used to assess the association between independent variables (physical activity, BMI, area of residence) and dietary patterns. Each model was mutually adjusted for confounding factors. Factor scores (continuous variables) of food patterns were the dependent variables. Linear regression analysis was carried out to determine the percentage variation in dietary-pattern scores, physical activity, BMI and body fat as explained by area of residence (urban vs. rural).

\section{Results}

The girls in the study $(n=552)$ were of low socio economic status with a mean monthly family income of 106 
USD. Of the participants $7.3 \%$ had a monthly income below the official poverty line ( $<24 \mathrm{USD} /$ month) [15]. Categorized by residential status; urban girls $(n=262)$ had a significantly $(P<0.005)$ higher percentage body fat (urban: $21.7 \pm 9.03$ vs. rural: $18.1 \pm 7.4$ ), WC (urban: 66.7 $\pm 8.9 \mathrm{~cm}$ vs. rural: $65.4 \pm 7.1 \mathrm{~cm}$ ) and BMI (urban: 19.1 $\pm 3.7 \mathrm{~kg} / \mathrm{m}^{2}$ vs. rural: $18.5 \pm 2.9 \mathrm{~kg} / \mathrm{m}^{2}$ ), than the rural girls $(n=292)$. When body composition (controlled for physical activity) was assessed according to the area of residence, an urban girl had three times greater likelihood of having excess body fat than a rural girl $(P=0.01, \mathrm{OR}=3.2)$. Time spent on physical activity was significantly $(P=0.003)$ higher among the rural girls (urban: $19.7 \pm 13.6 \mathrm{hrs} /$ week vs. rural: $22.9 \pm 11.9 \mathrm{hrs} /$ week) (Table 1 ).

\section{Dietary pattern analysis}

The KMO $(0.836)$ and BTS tests $(P<0.01)$ showed that the data was adequate for factor analysis. Two patterns were identified; together they explained $34.9 \%$ of the dietary intake variance. The convenience based dietary pattern explained $24.6 \%$ of the model variance and was characterized by high intakes of wheat flour based products, pulses, fruits, sugary foods, sugary beverages, fried foods, dairy products and animal food. The traditional dietary pattern explained $10.3 \%$ of the model variance and was characterized by high intakes of rice and rice based products, potatoes and tubers, vegetables and dark green leafy vegetables. All food groups had positive saturation in at least one of the two dietary patterns retained. Only 'liver' had communalities below 0.2 , and was removed from the model as it was not representative of the local culture and it did not help differentiate the dietary patterns identified (Table 2). Following PCA analysis, two dietary patterns were identified for the adolescent girls under study. While the mean factor score for convenience based dietary-pattern was significantly higher in the urban girls, the score for the traditional

Table 1 Characteristics of the study population

\begin{tabular}{lrrlll}
\hline & Urban & Rural & $\begin{array}{l}\text { Odds } \\
\text { ratio } \\
(\operatorname{EXP}(\beta))\end{array}$ & $P$ & $95 \% \mathrm{Cl}$ \\
\hline Body fat & $21.7 \pm 9.03^{1}$ & $18.1 \pm 7.4$ & $3.20^{*}$ & 0.01 & $1.3-7.9$ \\
Pattern 1 & $0.378 \pm 1.0^{2}$ & $-0.326 \pm 0.8$ & $0.708^{* *}$ & $<0.001$ & $0.548-$ \\
& & & & 0.867 \\
Pattern 2 & $-0.44 \pm 0.9$ & $0.38 \pm 0.94^{3}$ & $0.391^{* *}$ & $<0.019$ & $0.065-$ \\
& & & & & 0.718 \\
$\begin{array}{l}\text { Physical } \\
\text { activity } \\
\text { (hrs/wk) }\end{array}$ & $19.7 \pm 13.6$ & $22.9 \pm 11.9^{4}$ & & & \\
\hline
\end{tabular}

*Reference: Urban, controlled for physical activity and BMI

**Reference: Urban, controlled for physical activity

${ }^{1,2}$, Significantly higher among urban girls; ${ }^{1} 0 ., P=0.005,{ }^{2} P<0.001$

${ }^{4}$ Significantly higher among rural girls; $(P<0.001)$ dietary-pattern was significantly higher in the rural girls. Scores for the convenience-based dietary pattern were significantly $(P<0.005)$ higher for overweight girls and girls with low physical activity (Table 3 ).

Considering the percentage of variation as determined by area of residence; $29.7 \%$ of variation in dietary score was determined by area of residence $(12.3 \%$ by pattern 1 and $17.4 \%$ by pattern 2), $2 \%$ variation in BMI was determined by dietary patterns, and $6.2 \%$ variation in body fat was determined by dietary patterns.

When controlled for both BMI and physical activity, a girl residing in the urban area has a 0.708 likelihood of consuming a convenience-based dietary-pattern compared to a rural girl $(P<0.001 ; 95 \% \mathrm{CI}=0.548-0.867)$.In both urban and rural girls, when controlled for physical activity, a girl consuming a traditional dietary pattern showed a trend towards a 0.391 times likelihood of not being overweight $(P=0.019 ; 95 \% \mathrm{CI}=0.065-0.718)$.

On analyzing the focus group discussion data, urban and rural girls had similarities as well as differences in their practices and perceived constraints. Urban girls preferred to purchase meals from food outlets/vendors to save time, money (as cooking fuel for urban girls was costly). Conversely the rural girls consumed a home cooked meal, mainly consisting of rice and vegetables. Even though the rural areas are abundant in fruit, the rural girls were in the habit of selling their home garden produce as a source of income (Table 4).

\section{Discussion}

Our results, demonstrate a significant association between urban or rural living and body composition, namely body weight and fat. We identified two main dietary patterns in this population; a convenience based dietary pattern associated with urban dwelling, low physical activity and unhealthy body composition, and a traditional dietary pattern associated with rural dwelling, higher physical activity and healthy body composition. While all participants under study had similar socio economic status and education levels, the area of residence was associated with their life style and their body composition.

Our PCA analysis has been strengthened by the results of the focus group discussions, which have enriched the evidence provided by the quantitative data.

Negative effects of urbanization on life style and health are widely acknowledged, with urbanization shown to be linked to higher body-fat. [3, 16, 17]. Our results indicate that girls with high body fat and BMI followed the convenience based pattern and that an urban girl had a three times greater likelihood of having a high percentage body fat than a rural girl. Urban girls also had a greater BMI and waist circumference. The level of physical activity too was significantly lower among urban girls. Rural girls engaged in more physical activity, resulting in 
Table 2 Factor loadings and communalities estimated for the identified dietary patterns

\begin{tabular}{|c|c|c|c|}
\hline & \multicolumn{2}{|c|}{ Factor loading } & \multirow[t]{2}{*}{ Communalities } \\
\hline & $\begin{array}{l}\text { Pattern- } \\
1\end{array}$ & $\begin{array}{l}\text { Pattern- } \\
2\end{array}$ & \\
\hline Rice & & .716 & 0.606 \\
\hline Potatoes and Tubers & & .554 & 0.331 \\
\hline Vegetables & & .424 & 0.353 \\
\hline DGLV & & .339 & 0.223 \\
\hline Other starch (excluding rice) & .637 & & 0.620 \\
\hline Pulses & .376 & & 0.213 \\
\hline Fresh fruits (including pickle) & .709 & & 0.531 \\
\hline $\begin{array}{l}\text { Fruits with added sugar (i.e. fruit } \\
\text { salad) }\end{array}$ & .639 & & 0.417 \\
\hline Fish & .517 & & 0.353 \\
\hline Meat & .490 & & 0.324 \\
\hline Processed meat & .512 & & 0.299 \\
\hline Egg & .433 & & 0.256 \\
\hline Dairy & .645 & & 0.421 \\
\hline Sweetened beverages & .457 & & 0.209 \\
\hline Sweets & .532 & & 0.300 \\
\hline Fried food & .602 & & 0.374 \\
\hline Eigenvalues & 4.2 & 1.7 & \\
\hline$\%$ of explained variance & 24.6 & 10.3 & \\
\hline $\begin{array}{l}\% \text { of accumulated explained } \\
\text { variance }\end{array}$ & 24.6 & 34.9 & \\
\hline
\end{tabular}

lower likelihood of obesity. Evidence from elsewhere support our observation, with traditional diets being associated with normal weight among Brazilian school children [18].Chinese data indicate that high socio economic status (SES) was associated with high fat [19] and that cooking

Table 3 Comparison of factor scores of the two dietary patterns

\begin{tabular}{lrr}
\hline & Pattern-1 & \multicolumn{1}{c}{ Pattern-2 } \\
\hline Area of residence & & \\
Urban $(n=261)$ & $0.378 \pm 1.0^{1}$ & $-0.44 \pm 0.9$ \\
Rural $(n=298)$ & $-0.326 \pm 0.8$ & $0.38 \pm 0.9^{2}$ \\
BMl status* & & \\
Underweight & $-0.016 \pm 1.0$ & $0.08 \pm 0.8$ \\
Normal weight & $-0.003 \pm 0.9$ & $-0.013 \pm 1.0$ \\
Overweight & $0.16 \pm 1.0^{3}$ & $-0.34 \pm 0.9$ \\
Physical activity & & \\
Low $(<9$ hrs/wk) $(n=110)$ & $0.07 \pm 0.9^{1}$ & $-0.3 \pm 0.9$ \\
Medium ( $9-34$ hrs/wk) $(n=346)$ & $-0.07 \pm 1.0$ & $-0.01 \pm 0.9$ \\
High ( $>34$ hrs/wk) $(n=101)$ & $0.17 \pm 0.9$ & $0.35 \pm 1.0$ \\
\hline
\end{tabular}

*age specific World Health Organisation (WHO) cut off values for Body Mass Index (BMI) (de Onis et al., 2007)

${ }_{1,3}$ Significantly higher scores for pattern $\left.1 ;{ }^{1}(P<0.001),\right)_{1}^{3}(P=0.037)$

${ }^{2}$ Significantly higher scores for pattern $2 ;(P<0.001)$ patterns changed from high-carbohydrate foods toward high-fat, high-energy density foods. However our study participants were from a low SES back ground and the preference for purchase of food from vendors rather than a home cooked meal was attributed to limited cooking space in their homes and higher costs associated with having to purchase cooking fuel, as opposed to their rural counterparts who were able to obtain firewood freely. The observed differences in dietary patterns can be attributed to urbanization as witnessed by the link to not only urban residence, but also urban driven behaviour.

The qualitative component of our data identify lifestyle factors associated with urban versus rural living and give greater depth to the understanding of reasons for these differences which influence dietary patterns.

The dietary intake variance explained by our analysis was similar to or better than the variance reported for similar studies on other populations. While pattern-1 explained $24.6 \%$ of the model variance and Pattern- 2 explained $10.3 \%$ of the model variance. Identified together the two patterns explain $34.9 \%$ of the dietary intake variance.

A cross sectional school based study of Brazilian adolescents between 14 and 19 years identified three dietary patterns which together explained $35.3 \%$ of the dietary intake variance [18]. Similarly a dietary pattern analysis conducted on children from the Mysore Parthenon study, identified three patterns with $9.1 \%, 7.5 \%$ and $4.1 \%$ variance, where the total dietary intake variance explained was $20.7 \%$ [4].

Our results, when compared with those of other countries in transition show that the food categories (i.e. fried food, sweetened food) within the convenience-based dietary-pattern are similar although individual foods differ as would be expected since they are likely to be country or region specific.

While most patterning studies tend to identify western, mixed and traditional patterns [18, 20],we identified a convenience based dietary pattern supported by the qualitative data, that is high in non rice starches, animal food, dairy, sugar and fat and rural girls report higher consumption of rice and lower consumption of animal products. The rural girl who had more access to home grown produce indicated a preference for home cooked meals. Therefore it is most likely that, in our population, the transition from the traditional rice based diet to the urban diet appears to be driven by convenience, necessities and constraints of urban living including lack of space and cost. This is also apparent by the mixed variety of foods; fried or sweetened food, sweetened beverages and starch which are available in an urban environment with cost and preference being influencing factors .Fruit consumption was also seen among urban dwellers as fruits could be bought in a ready to eat form (in the form of cut fruit in sugar syrup or salt and chili), and highlight the availability factor influencing purchase of healthful foods. 
Table 4 Focus Group Discussion data (Qualitative data)

\begin{tabular}{|c|c|c|}
\hline Theme & Urban & Rural \\
\hline \multirow[t]{6}{*}{ Practices } & $\begin{array}{l}\text { - Higher consumption of: } \\
\text { processed food, animal food, } \\
\text { bread, deep fried food. }\end{array}$ & $\begin{array}{l}\text { - Higher consumption of: } \\
\text { fruits, dark green leafy } \\
\text { vegetables, rice and rice } \\
\text { based products }\end{array}$ \\
\hline & \multirow{5}{*}{$\begin{array}{l}\text { - Concept of cost } \\
\text { effectiveness - cheaper to } \\
\text { buy due to minimum } \\
\text { preparation cost, hence food } \\
\text { is bought from food outlets/ } \\
\text { vendors }\end{array}$} & $\begin{array}{l}\text { - Frequent consumption of } \\
\text { tea (even after meals) }\end{array}$ \\
\hline & & $\begin{array}{l}\text { - Most meals were home } \\
\text { cooked and vegetarian }\end{array}$ \\
\hline & & $\begin{array}{l}\text { - Use of greater quantity of } \\
\text { coconut milk in cooking }\end{array}$ \\
\hline & & $\begin{array}{l}\text { - Fish / meat was } \\
\text { consumed occasionally } \\
\text { (once/ week) }\end{array}$ \\
\hline & & $\begin{array}{l}\text { - Selling of fruits from their } \\
\text { gardens (eg. Papaya) in } \\
\text { exchange for imported } \\
\text { fruits such as apples, } \\
\text { oranges }\end{array}$ \\
\hline \multirow[t]{3}{*}{$\begin{array}{l}\text { Perceived } \\
\text { Constraints }\end{array}$} & $\begin{array}{l}\text { - Cooking at home is time } \\
\text { consuming }\end{array}$ & $\begin{array}{l}\text { - Animal food is too } \\
\text { expensive }\end{array}$ \\
\hline & - Cooking at home is costly & \multirow{2}{*}{$\begin{array}{l}\text { - Attaching "value" to } \\
\text { imported and expensive } \\
\text { fruits }\end{array}$} \\
\hline & $\begin{array}{l}\text { - Instant food is cheaper than } \\
\text { rice and curry }\end{array}$ & \\
\hline Similarities & \multicolumn{2}{|c|}{$\begin{array}{l}\text { Poor time and financial management ; Cooking: mainly by } \\
\text { mothers / unless married }\end{array}$} \\
\hline
\end{tabular}

While nutrition transition is influenced by availability of high energy foods at low cost [6], our results indicate that if healthy options are available and reasonably priced, consumption maybe expected. Unexpectedly, the traditional pattern associated with the rural girls had a lower factor loading for fruits than the convenience-based dietarypattern. However, insight from focus groups indicate that fruits grown in rural home gardens are mostly sold, and what is eaten are imported fruits, which being expensive compared to local fruits are consumed rarely and in smaller portion sizes. The importance of value addition to healthy foods therefore becomes relevant to both rural and urban environments.

The use of questionnaires to obtain dietary data could be considered a limitation in this study due to the known inherent possibility of recall bias as must be born in mind when interpreting the results. However in this study the use of focus group data which corroborate the findings of the dietary pattern analysis adds strength to the overall validity of the results. The limitation of using skin fold thickness for the assessment of body fat has been addressed to some extent through the use of an equation specific for 15 - 19 year old Sri Lankan adolescent girls in the calculation of body fat.

\section{Conclusions}

The study concludes that urbanization is associated with the adolescent girls practices, diet and thereby her body composition. While a traditional dietary pattern was associated with a rural setting along with higher physical activity and favorable body composition urbanization has led to a convenience based dietary pattern, lower physical activity and adverse body composition. Dietary convenience is a vital factor that needs to be considered along with urbanization, the importance of healthy snacking can be one successful intervention option for urban areas, with value addition to traditional foods being essential and relevant to both environments.

\section{Abbreviations}

BTS: Bartlett test of sphericity; BMI: Body Mass Index; DGLV: Dark Green Leafy Vegetables; KMO: Kaiser-Meyer-Olkin; PCA: Principal Component Analysis; PHM: Public Health Midwife; SFT: Skin-Fold Thickness; SES: Socio Economic Status; WHO: World Health Organisation.

\section{Competing interests}

No competing interests.

\section{Authors' contributions}

$\mathrm{PL}$, AdeS and SA designed research; MD conducted research; MD performed statistical analysis; MD, PL and AdeS wrote paper; AdeS had primary responsibility for final content. All authors have read and approved the final manuscript.

\section{Acknowledgments}

The authors acknowledge all study participants and their families for their cooperation and the

Public health Midwives of the Medical officer of health $(\mathrm{MOH})$ areas of the Colombo and Kalutara districts for coordinating with the participants and providing space to carry out the investigations in the $\mathrm{MOH}$ officers. The International Atomic Energy Agency (IAEA)Vienna, Austria,(SRL6/030) and the Nutrition Coordination Division Sri Lanka (2.1.b.G/vi), for funding this study.

\section{Author details}

${ }^{1}$ Department of Biochemistry and Molecular Biology, Faculty of Medicine, University of Colombo, Kynsey road, Colombo 8, Sri Lanka. Department of Physiology, Faculty of Medicine, University of Colombo, Kynsey road, Colombo 8, Sri Lanka.

Received: 27 March 2015 Accepted: 26 October 2015

Published online: 03 November 2015

\section{References}

1. Popkin BM. The nutrition transition and obesity in the developing world. J Nutr. 2001;131:871S-3S.

2. Popkin BM. The nutrition transition in low-income countries: an emerging crisis. Nutr Rev. 1994;52:9.

3. Popkin BM. Urbanization, Lifestyle Changes and the Nutrition Transition. World Dev. 1999:27(11):1905-16.

4. Kehoe SH, Krishnaveni GV, Veena SR, Guntupalli AM, Margetts BM, Fall CHD, et al. Diet patterns are associated with demographic factors and nutritional status in South Indian children. Maternal Child Nutr. 2014;10(1):145-58.

5. Lioret S, Touvier M, Lafay L, Volatier JL, Maire B. Dietary and physical activity patterns in French children are related to overweight and socio economic status. J Nutr. 2008;138:101-7.

6. Johnson L, Mander AP, Jones LR, Emmett PM, Jebb SA. Energy-dense, lowfiber, high-fat dietary pattern is associated with increased fatness in childhood. Am J Clin Nutr. 2008:87:846-54.

7. Ritchie LD, Spector P, Stevens MJ, Schmidt MM, Schreiber GB, StriegelMoore $\mathrm{RH}$, et al. Dietary patterns in adolescence are related to adiposity in young adulthood in black and white females. J Nutr. 2007;137(2):399-406.

8. Maithili Band Saxena V. Adolescent's educational status and reasons for dropout from the school. Indian J Community Med. 2008;33(2):127-8.

9. Lanerolle P, Atukorala S. Iron and vitamin A status of adolescent school girls in an urban and a rural area of Sri Lanka. Ceylon J Med Sci. 1998;41(2):35-45. 
10. de Lanerolle-Dias M, de Silva A, Lanerolle P, Arambepola C, Atukorala S. Micronutrient status of female adolescent school dropouts. Ceylon Med J. 2012;57:74-8.

11. de Ones M, Onyango AW, Borghi E, Siyam A, Nishida C, Siekmann J. Development of a WHO growth reference for school-aged children and adolescents. Bull World Health Organ. 2007;85:660-7.

12. de Lanerolle-Dias M, de Silva A, Lanerolle P, Arambepola C, Atukorala S. Body fat assessment in Sri Lankan adolescent girls; development of a simple field tool. Ann Hum Biol. 2011;38(3):330-6.

13. Stewart DW, Shamdasani PN. Focus groups: Theory and practice. London: Sage publications; 1993.

14. Basilevsky AT. Statistical factor analysis and related methods -theory and application. New York: Wiley Interscience; 1994

15. Sri Lanka Labour Force Survey Annual Report Summary Statistics on Labour Force Characteristics. Department of Census and Statistics, Ministry of Finance and Planning, Sri Lanka; 2009.

16. Christensen DL, Eis J, Hansen AW, Mwaniki DL, Kilonzo B, et al. Obesity and regional fat distribution in Kenyan populations: Impact of ethnicity and urbanization. Ann Hum Biol. 2008;35(2):232-49.

17. Ekezie J, Anyanwu EG, Danborno B, Anthony U. Impact of urbanization on obesity, anthropometric profile and blood pressure in the Igbos of Nigeria. North Am J Med Sci. 2011;3(5):242-6.

18. Rodrigues PRM, Pereira RA, Cunha DB, Sichieri R, Ferreira MG, Vilela AA, et al. Factors associated with dietary patterns in adolescents: A school-based study in Cuiabá, Mato Grosso. Braz J Epidemiol. 2012;15(3):662-74.

19. Du S, Mroz TA, Zhai F, Popkin BM. Rapid income growth adversely affects diet quality in China particularly for the poor. Soc Sci Med. 2004;59(7):150515.

20. Garduno-Diaz SD, Khokhar S. South Asian dietary patterns and their association with risk factors for the metabolic syndrome. J Hum Nutr Diet. 2013;26(2):145-55.

\section{Submit your next manuscript to BioMed Central and take full advantage of:}

- Convenient online submission

- Thorough peer review

- No space constraints or color figure charges

- Immediate publication on acceptance

- Inclusion in PubMed, CAS, Scopus and Google Scholar

- Research which is freely available for redistribution 